\title{
Trastuzumab for Early Breast Cancer: Hope or Hype?
}

\author{
Ian E. Smith \\ Royal Marsden Hospital, London, UK
}

Trastuzumab (Herceptin ${ }^{\circledR}$, Hoffmann-La Roche, GrenzachWyhlen, Germany) is a humanised monoclonal antibody developed against the HER2 transmembrane growth factor receptor. HER 2 over-expression occurs in around $20 \%$ of women with early breast cancer and is associated with an adverse prognosis. A few years ago Herceptin was shown to be active as a single agent against metastatic breast cancer and to improve survival markedly when given in combination with chemotherapy. Based on these results, 4 large randomised adjuvant Herceptin trials were set up around the world. First results were announced recently and have created a great wave of publicity and controversy. On the one hand they have been welcomed with excitement and enthusiasm by breast cancer specialists everywhere and hailed by an international expert as 'simply stunning' [1]. On the other hand they have been questioned by public health specialists and criticised by a leading medical journal as being 'premature' and 'insufficient to make reliable judgements' [2]. How are the public and in particular patients with breast cancer to judge these widely differing views?

The background to this debate is as follows. The 4 trials involve more than 13,000 high-risk women with HER2-positive early breast cancer, most but not all of whom had axillary lymph node involvement. All the trials used Herceptin for one year but given in slightly different ways. All of them have shown in their first planned analysis that Herceptin given with or after chemotherapy reduces the early risk of recurrence by around $50 \%$. The 2 USA-based trials (NSAPBB-B31 and NCCTGN-9831) have amalgamated their data and showed a Herceptin benefit in combination with paclitaxel chemotherapy following anthracycline treatment [3]. The HERA trial, based worldwide apart from the USA, showed similar efficacy with Herceptin given as single agent treatment after standard chemotherapy [4]. The BCIRG-006 trial, again worldwide, showed a benefit with docetaxel after anthracyclines, confirming the results of the American trials, but also showed benefit with a novel non-anthracycline schedule using front-line Herceptin in combination with docetaxel and carboplatin [5]. Even at this early stage the combined American analysis is already showing a significant survival benefit and strong trends towards improved survival are emerging in the others. In practical terms these results mean on average a $20 \%$ absolute improvement in the predicted 4-year survival with Herceptin treatment [3] and for those patients at highest risk the absolute gain will probably be greater.

Breast cancer specialists see these results as unprecedented; when they were presented for the first time early last summer they were greeted with a gasp of surprise and prolonged applause from the thousands of delegates in the audience. Nevertheless the criticism that the results are premature must be taken seriously. There are many examples elsewhere in cancer medicine of promising early results failing the test of time. Here, however, we believe that long-term confidence is justified for two very good reasons: first, the trials are very large with enormous statistical power; the $\mathrm{p}$ value on the combined USA data was $3 \times 10^{-12}$, and all 4 trials showed very similar results. Second, the simple fact is that twenty years of experience in adjuvant breast cancer trials for both endocrine therapy and chemotherapy have consistently shown that early benefits are sustained with time and indeed often improved [6]. This is of course not a guarantee that the same will be true for Herceptin, but those of us who treat breast cancer believe that the magnitude of the early benefit and the great weight of past evidence justifies our optimism and enthusiasm.

The other main criticism of these trials concerns safety issues. In the early trials in metastatic disease, Herceptin quite unexpectedly was found to be associated with an increased risk of cardiotoxicity, particularly when given in combination with or shortly after anthracyclines. The adjuvant trials were therefore designed to give Herceptin after rather than with anthracyclines. Despite this the B-31 trial has shown an increase in

\begin{tabular}{ll}
\hline KARGER & ๑ 2006 S. Karger GmbH, Freiburg \\
Fax +49 7614520714 & Accessible online at: \\
$\begin{array}{l}\text { E-mail Information@Karger.de } \\
\text { www.karger.com }\end{array}$ & www.karger.com/brc
\end{tabular}


cardiotoxicity with Herceptin but it is small: the cumulative 3 year cardiotoxicity was $3.3 \%$ greater than in the control arm [7]. The incidence of cardiac failure in the HERA trial (with a longer interval between anthracycline chemotherapy and Herceptin) was $1.7 \%$ including only $0.5 \%$ for severe failure. Furthermore Herceptin-induced cardiac failure usually responds well to treatment [8] and so far there has only been one Herceptin-related cardiac death in all 4 trials. Many thousands of additional women have received Herceptin over the last 10 years for metastatic breast cancer and no other significant toxicities have emerged. Careful further observation is essential for all patients in these trials, but the argument that adjuvant Herceptin should not yet be made readily available because of toxicity fears is unwarranted; the evidence at present shows that the potential benefits far outweigh the risks.

Everyone must keep an open mind as results mature and many questions remain unanswered. The best schedule and the optimal duration of treatment have yet to be determined. The HERA trial is assessing 2 years of treatment as well as 1, and in contrast a small trial from Finland has suggested provocatively that very short duration Herceptin may also be very effective. The results relate only to women with high-risk cancers also requiring chemotherapy; there is no good evidence for benefit in low-risk women. The treatment is undeniably expensive, but the cost of treating recurrent breast cancer is even more so, and the prevention of relapse should lead to eventual savings. These issues will take a long time to resolve. Meanwhile breast cancer specialists around the world believe that women with high-risk HER2-positive breast cancer should be fully informed about the early Herceptin results, with appropriate caution on the absence of long-term data. On simple humanitarian grounds such information must also be accompanied by the right to have access to this treatment now, should they so choose.

\section{References}

1 Hortobagyi GN: Trastuzumab in the treatment of breast cancer. New Engl J Med 2005;353. 1734-1736.

2 Horton R: Herceptin and early breast cancer: a moment for caution. Lancet 2005;366;1673.

3 Romond EH, Perez EA, Bryant J, Suman VJ, Geyer CE, Davidson NE, Tan-Chiu E, Martino S, Paik S, Kaufman PA, Swain SM, Pisansky TM, Fehrenbacher L, Kutteh LA, Vogel VG, Visscher DW, Yothers G, Jenkins RB, Brown AM, Dakhil SR, Mamounas EP, Lingle WL, Klein PM, Ingle JN, Wolmark N: Trastuzumab plus adjuvant chemotherapy for operable HER2-positive breast cancer. New Engl J Med 2005;353:1673-1684.
4 Piccart-Gebhart MJ, Procter M, Leyland-Jones B, Goldhirsch A, Untch M, Smith I, Gianni L, Baselga J, Bell R, Jackisch C, Cameron D, Dowsett M, Barrios $\mathrm{CH}$, Steger G, Huang C-S, Andersson M, Inbar M, Lichinitser M, Láng I, Nitz U, Iwata $H$, Thomssen C, Lohrisch C, Suter TM, Rüschoff J, Sütö T, Greatorex V, Ward C, Straehle C, McFadden E, Dolci S, Gelber RD, for the Herceptin Adjuvant (HERA) Trial Study Team: Trastuzumab after adjuvant chemotherapy in her2-positive breast cancer. New Engl J Med 2005;353: 1659-1672.

5 Breast Cancer International Research Group (BCIRG): Interim analysis of phase III study shows docetaxel-based chemotherapy regimens combined with trastuzumab significantly improved disease free survival in early stage Her2 positive breast cancer. Proceedings from the 28th annual San Antonio Breast Cancer Symposium. December 2005. Abstr 1
6 Early Breast Cancer Trialists Collaborative Group (EBCTCG): Effects of chemotherapy and hormonal therapy for early breast cancer on recurrence and 15-year survival: an overview of the randomised trials. Lancet 2005;365;1687-1717.

7 Tan-Chiu E, Yothers G, Romond R, Geyer CE, Ewer M, Keefe D, Shannon RP, Swain SM, Brown A, Fehrenbacher L, Vogel VG, Seay TE, Rastogi P, Mamounas EP, Wolmark N, Bryant J: Assessmen of cardiac dysfunction in a randomized trial comparing doxorubicin and cyclophosphamide followed by paclitaxel, with or without trastuzumab as adjuvant therapy in node-positive, human epidermal growth factor receptor 2-overexpressing breast cancer. NSABP B-31. J Clin Oncol 2005;23: 7811-7819.

8 Ewer MS, Vooletich MT, Durand JB, Woods ML, Davis JR, Valero V, Lenihan DJ: Reversibility of trastuzumab-related cardiotoxicity: new insights based on clinical course and response to medical treatment. J Clin Oncol 2005;23:7820-7826. 